MATHEMATICS OF COMPUTATION

Volume 74, Number 252, Pages 1621-1636

S 0025-5718(05)01756-4

Article electronically published on March 18, 2005

\title{
SUPERCONVERGENCE OF SPECTRAL COLLOCATION AND $p$-VERSION METHODS IN ONE DIMENSIONAL PROBLEMS
}

\author{
ZHIMIN ZHANG
}

\begin{abstract}
Superconvergence phenomenon of the Legendre spectral collocation method and the $p$-version finite element method is discussed under the one dimensional setting. For a class of functions that satisfy a regularity condition (M): $\left\|u^{(k)}\right\|_{L^{\infty}} \leq c M^{k}$ on a bounded domain, it is demonstrated, both theoretically and numerically, that the optimal convergent rate is supergeometric. Furthermore, at proper Gaussian points or Lobatto points, the rate of convergence may gain one or two orders of the polynomial degree.
\end{abstract}

\section{INTRODUCTION}

Perhaps the most appreciated property of the spectral method/ $p$-version (finite element) method is the spectral accuracy, geometric/exponential convergent rate. This remarkable behavior is well understood [3, 5, 6, 8, 9, 13, 16, 19, 21. In the literature, some researchers observed supergeometric convergence rate (see, e.g., 18) in numerical tests using spectral collocation methods. However, a theoretical justification of this phenomenon is lacking.

Observation from interpolation. Let $L_{k}$ be the Legendre polynomial of degree $k$ with $L_{k}(1)=1$ and define

$$
\phi_{k+1}(x)=\int_{-1}^{x} L_{k}(t) d t, \quad k=1,2, \ldots
$$

The following properties are valid:

$$
\phi_{k+1}(x)=\frac{1}{2 k+1}\left(L_{k+1}(x)-L_{k-1}(x)\right)=\frac{1}{k(k+1)}\left(x^{2}-1\right) L_{k}^{\prime}(x) .
$$

Zeros of $\phi_{k}$ are called Gauss-Lobatto points of degree $k$. If $f_{p} \in \mathcal{P}_{p}[-1,1]$ interpolates a continuous function $f$ at the $p+1$ Gauss-Lobatto points $-1=x_{0}<x_{1}<$ $\cdots<x_{p}=1$, then the remainder of the interpolation is

$$
\begin{aligned}
f(x)-f_{p}(x) & =f\left[x_{0}, x_{1}, \ldots, x_{p}, x\right] c_{1}(p)(2 p+1) \phi_{p+1}(x) \\
& =f\left[x_{0}, x_{1}, \ldots, x_{p}, x\right] c_{1}(p)\left(L_{p+1}(x)-L_{p-1}(x)\right),
\end{aligned}
$$

Received by the editor April 28, 2004 and, in revised form, July 16, 2004.

2000 Mathematics Subject Classification. Primary 65N30, 65N15.

Key words and phrases. Spectral collocation method, $p$-version finite element method, exponential rate of convergence, superconvergence.

This work was supported in part by the National Science Foundation grants DMS-0074301 and DMS-0311807. 
with 22 ]

$$
c_{1}(p)=2^{p+1} /\left(\begin{array}{c}
2 p+2 \\
p+1
\end{array}\right) \approx \frac{\sqrt{\pi(p+1)}}{2^{p+1}} .
$$

When $f \in C^{p+1}[-1,1]$, the divided difference

$$
f\left[x_{0}, x_{1}, \ldots, x_{p}, x\right]=\frac{f^{(p+1)}\left(\xi_{x}\right)}{(p+1) !}, \quad \xi_{x} \in(0,1) .
$$

Furthermore, if $f$ satisfies condition $(\mathrm{M})$, we have the estimate

$$
\left\|f-f_{p}\right\|_{L^{\infty}[-1,1]} \leq c_{1}(p) \frac{2 c M^{p+1}}{(p+1) !} \leq C\left(\frac{e M}{2(p+1)}\right)^{p+1},
$$

by the Stirling formula (A.2). Here and in the rest of this paper, $C$ stands for a generic constant, which is not necessary the same in different places.

We may also consider the remainder for the derivative

$$
\begin{aligned}
f^{\prime}(x)-f_{p}^{\prime}(x)= & f\left[x_{0}, x_{1}, \ldots, x_{p}, x, x\right] c_{1}(p)\left(L_{p+1}(x)-L_{p-1}(x)\right) \\
& +f\left[x_{0}, x_{1}, \ldots, x_{p}, x\right] c_{1}(p)(2 p+1) L_{p}(x)
\end{aligned}
$$

and to obtain the estimate

$$
\left\|f^{\prime}-f_{p}^{\prime}\right\|_{L^{\infty}[-1,1]} \leq c_{1}(p) \frac{2 c M^{p+2}}{(p+2) !}+c_{1}(p)(2 p+1) \frac{c M^{p+1}}{(p+1) !} \leq C\left(\frac{e M}{2(p+1)}\right)^{p} .
$$

Comparing (1.4) and (1.5), we see that the error for the function value approximation is better than that of the derivative by a factor $p+1$. However, if evaluating the derivative errors at the Gaussian points $g_{j}$, i.e., zeros of $L_{p}(x)$, we obtain

$$
\left|\left(f^{\prime}-f_{p}^{\prime}\right)\left(g_{j}\right)\right| \leq c_{1}(p) \frac{2 c M^{p+2}}{(p+2) !} \leq C\left(\frac{e M}{2(p+1)}\right)^{p+2} .
$$

We see that at the Gaussian points, the approximation error for the derivative is improved from the global one by a factor $(p+1)^{2}$. This motivates the following definition.

Definition. The error $e_{p}=u-u_{p}$ is said to have superconvergence at a set of points $\left\{\xi_{p, j}\right\}$ with order $\gamma>0$ if there exists a constant $a>0$ such that

$$
\lim _{p \rightarrow \infty} \frac{\left\|e_{p}\right\|_{L^{\infty}[-1,1]}}{p^{\gamma} \max _{j}\left|e_{p}\left(\xi_{p, j}\right)\right|} \rightarrow a .
$$

We observe the following from the above discussion.

(1) All three error estimates (1.4)-(1.6) are supergeometric in the sense

$$
\exp (p(\kappa-\log p)), \quad \kappa=\log \frac{e M}{2},
$$

which is better than the usual spectral convergent rate $\exp (-\sigma p)$ for analytic functions.

(2) At the Gaussian points, the derivative approximation error is superconvergent.

Now the question is whether we are able to realize (1) and (2) with the $p$-version finite element and spectral collocation methods in solving differential equations. The current paper provides an affirmative answer to this question. We shall prove, using a simple model with favorable condition, that (1) and (2) are indeed the case. In addition, we demonstrate, by numerical examples, that our estimates are sharp. 
We end this section by listing some properties of $L_{j}$ and $\phi_{j}$ which will be used later:

$$
\begin{gathered}
\left\|L_{j}\right\|_{L^{2}[-1,1]}^{2}=\frac{2}{2 j+1}, \quad\left\|\phi_{j}\right\|_{L^{2}[-1,1]}^{2}=\frac{4}{(2 j-3)(2 j-1)(2 j+1)}, \\
\left\|L_{p+1}-L_{p-1}\right\|_{L^{2}[-1,1]}^{2}=\frac{4(2 p+1)}{(2 p-1)(2 p+3)}, \\
\sum_{j=2}^{p} \frac{(2 j-1)^{2}}{4}\left\|\phi_{j}\right\|_{L^{2}[-1,1]}^{2}\left\|L_{j-1}\right\|_{L^{2}[-1,1]}^{2}=\frac{2}{3}-\frac{2 p}{4 p^{2}-1} .
\end{gathered}
$$

Their proofs are straightforward and we only verify the last one. In fact, using (1.7), the left-hand side of (1.9) equals

$$
\sum_{j=2}^{p} \frac{2}{(2 j-3)(2 j+1)}=\frac{1}{2} \sum_{j=2}^{p}\left(\frac{1}{2 j-3}-\frac{1}{2 j+1}\right)=\frac{1}{2}\left(1+\frac{1}{3}-\frac{1}{2 p-1}-\frac{1}{2 p+1}\right)
$$

which equals the right-hand side of (1.9).

\section{The $p$-VERSiOn Finite ELEMENT METHOD}

We start from the simplest model

$$
-u^{\prime \prime}=f, \quad u(-1)=0=u(1)
$$

Its weak form is to find $u \in H_{0}^{1}[-1,1]$ such that

$$
\left(u^{\prime}, v^{\prime}\right)=(f, v)=\int_{-1}^{1} f(x) v(x) d x, \quad \forall v \in H_{0}^{1}[-1,1] .
$$

Due to this special feature, the solution has the expansion

$$
u(x)=\sum_{j=2}^{\infty} b_{j-1} \phi_{j}(x), \quad b_{j-1}=\frac{2 j-1}{2}\left(f, \phi_{j}\right),
$$

where $\phi_{j}$ is defined by (1.1). The $p$-version or Legendre spectral method is to find $u_{p} \in \mathcal{P}_{p} \cap H_{0}^{1}[-1,1]$ such that

$$
\left(u_{p}^{\prime}, \phi_{j}^{\prime}\right)=\left(f, \phi_{j}\right), \quad j=2, \ldots, p .
$$

Substituting $u_{p}(x)=\sum_{j=2}^{p} a_{j-1} \phi_{j}(x)$ into (2.4) and using (1.1) and (1.7), we obtain $a_{j}=b_{j}, j=1,2, \ldots, p-1$. Differentiating (2.3), we have

$$
u^{\prime}(x)=\sum_{k=1}^{\infty} b_{k} L_{k}(x)
$$

Therefore, the $b_{j}$ 's are coefficients of the Legendre expansion of $u^{\prime}$ with $b_{0}=0$, due to the boundary condition. 
Theorem 2.1. Let $u$ and $u_{p}$ be solutions of (2.2) and (2.4), respectively. Assume that $u$ satisfies condition $(\mathrm{M})$. Then when $(2 p+1)(2 p+3)>2 M^{2}$,

$$
\begin{aligned}
\left\|u^{\prime}-u_{p}^{\prime}\right\|_{L_{2}[-1,1]} & \leq C \sqrt{p}\left(\frac{e M}{2 p}\right)^{p+1}, \\
\left\|u-u_{p}\right\|_{L_{2}[-1,1]} & \leq \frac{C}{\sqrt{p}}\left(\frac{e M}{2 p}\right)^{p+1} .
\end{aligned}
$$

Here $C$ is independent of $p$ and $M$.

Proof. The error of the $(p-1)$-term Legendre expansion is

$$
\left\|u^{\prime}-u_{p}^{\prime}\right\|_{L_{2}[-1,1]}^{2}=\sum_{k=p}^{\infty} \frac{2}{2 k+1} b_{k}^{2}
$$

Using the result [14, p. 58, Theorem 2.1.6], we have

$$
b_{k}=\frac{2^{k} k !}{(2 k) !} u^{(k+1)}\left(\eta_{k}\right), \quad \eta_{k} \in(-1,1) .
$$

Note that $\frac{2^{k} k !}{(2 k) !}=\frac{1}{(2 k-1) ! !}$. Applying the regularity assumption $\left|u^{(k)}(x)\right| \leq c M^{k}$, we derive

$$
\begin{aligned}
\| u^{\prime} & -u_{p}^{\prime} \|_{L_{2}[-1,1]}^{2} \\
& <2\left(c M^{p+1}\right)^{2}\left(\frac{1}{(2 p-1) ! !(2 p+1) ! !}+\frac{M^{2}}{(2 p+1) ! !(2 p+3) ! !}+\frac{M^{4}}{(2 p+3) ! !(2 p+5) ! !}+\cdots\right) \\
& =\frac{2\left(c M^{p+1}\right)^{2}}{(2 p-1) ! !(2 p+1) ! !}\left(1+\frac{M^{2}}{(2 p+1)(2 p+3)}+\frac{M^{4}}{(2 p+1)(2 p+3)^{2}(2 p+5)}+\cdots\right) \\
& <\frac{4\left(c M^{p+1}\right)^{2}}{(2 p-1) ! !(2 p+1) ! !}
\end{aligned}
$$

when $(2 p+1)(2 p+3)>2 M^{2}$. This last term can be readily estimated by the Stirling type formula (A.1) - A.4

$$
(2 p-1) ! !(2 p+1) ! ! \quad \approx \frac{\sqrt{(2 p) !}}{\sqrt[4]{\pi(p+0.25)}} \frac{\sqrt{(2 p+2) !}}{\sqrt[4]{\pi(p+1.25)}} \approx \frac{(2 p) ! 2 p}{\sqrt{\pi p}} \approx\left(\frac{2 p}{e}\right)^{2 p} 2 \sqrt{2} p,
$$

which leads to (2.5) with $C=\sqrt[4]{2} c$. Under the asymptotic condition, the first term in the expansion (2.7) is the dominant term, which has the estimate

$$
\frac{2}{2 p+1} b_{p}^{2} \approx \frac{c^{2} p}{\sqrt{2}}\left(\frac{e M}{2 p}\right)^{2(p+1)} .
$$

Now we turn to estimating the error in the function value approximation,

$$
\begin{aligned}
\left(u-u_{p}\right)(x) & =\sum_{j=p}^{\infty} b_{j} \phi_{j+1}(x)=\sum_{j=p}^{\infty} \frac{b_{j}}{2 j+1}\left(L_{j+1}-L_{j-1}\right)(x) \\
& =-\frac{b_{p}}{2 p+1} L_{p-1}(x)-\frac{b_{p+1}}{2 p+3} L_{p}(x)+\sum_{j=p+1}^{\infty}\left(\frac{b_{j-1}}{2 j-1}-\frac{b_{j+1}}{2 j+3}\right) L_{j}(x) .
\end{aligned}
$$

To obtain

$$
\frac{b_{j-1}}{2 j-1}-\frac{b_{j+1}}{2 j+3}
$$


we compare the power series and the Legendre expansions of $u^{\prime}$,

$$
u^{\prime}(x)=\sum_{m=1}^{\infty} \frac{a_{m}}{m !} x^{m}=\sum_{i=1}^{\infty} b_{i} L_{i}(x) .
$$

Multiplying both sides by $L_{k}$ and integrating, we have

$$
\sum_{m=0}^{\infty} \frac{a_{m}}{m !} \int_{-1}^{1} x^{m} L_{k}(x) d x=\frac{2}{2 k+1} b_{k}
$$

Using the fact [15, p. $194(42)]$

$$
\frac{1}{2} \int_{-1}^{1} x^{m} L_{k}(x) d x=\frac{m !}{(m-k) ! !(m+k+1) ! !}, \quad m \geq k, m-k=0(\bmod 2),
$$

we obtain

$$
\begin{aligned}
b_{k} & =(2 k+1) \sum_{m \geq k, m-k=0(\bmod 2)} \frac{a_{m}}{(m-k) ! !(m+k+1) ! !} \\
& =(2 k+1)\left(\frac{a_{k}}{0 ! !(2 k+1) ! !}+\frac{a_{k+2}}{2 ! !(2 k+3) ! !}+\frac{a_{k+4}}{4 ! !(2 k+5) ! !}+\cdots\right) .
\end{aligned}
$$

Therefore,

$$
\frac{b_{j-1}}{2 j-1}-\frac{b_{j+1}}{2 j+3}=\frac{a_{j-1}}{(2 j-1) ! !}
$$

and hence,

$$
\left\|u-u_{p}\right\|_{L_{2}[-1,1]}^{2}=\frac{b_{p}^{2}}{(2 p+1)^{2}} \frac{2}{2 p-1}+\frac{b_{p+1}^{2}}{(2 p+3)^{2}} \frac{2}{2 p+1}+\sum_{j=p+1}^{\infty} \frac{a_{j-1}^{2}}{[(2 j-1) ! !]^{2}} \frac{2}{2 j+1} .
$$

Following similar arguments, it is straightforward to obtain the error bound (2.6) with the same constant $C$ as in (2.5).

Since $u_{p}$ is the Legendre expansion of $u$, the upper bound in Theorem 2.1 is also a lower bound under the nondegenerate assumption: $b_{p} \neq 0$.

Corollary 2.1. Under the nondegenerate assumption in addition to the hypotheses of Theorem 2.1, we have

$$
\begin{aligned}
& \left\|u-u_{p}\right\|_{L^{2}[-1,1]}=O\left[\frac{1}{\sqrt{p}}\left(\frac{e M}{2 p}\right)^{p+1}\right], \quad\left\|u^{\prime}-u_{p}^{\prime}\right\|_{L^{2}[-1,1]}=O\left[\sqrt{p}\left(\frac{e M}{2 p}\right)^{p+1}\right] \\
& \left\|u-u_{p}\right\|_{L^{\infty}[-1,1]}=O\left[\left(\frac{e M}{2 p}\right)^{p+1}\right], \quad\left\|u^{\prime}-u_{p}^{\prime}\right\|_{L^{\infty}[-1,1]}=O\left[p\left(\frac{e M}{2 p}\right)^{p+1}\right] .
\end{aligned}
$$

\section{Spectral COllocation Method}

In practice, collocation methods are usually employed. In our current situation, it is natural to collocate at the $p+1$ Gauss-Lobatto points. Therefore, we are seeking $w_{p} \in \mathcal{P}_{p} \cap H_{0}^{1}[-1,1]$ such that

$$
-w_{p}^{\prime \prime}\left(x_{j}\right)=f\left(x_{j}\right), \quad j=0,1, \ldots, p .
$$


A well-known fact is that the above collocation method is equivalent to a spectral method with numerical integration. Let $\omega_{j}, j=0,1, \ldots, p$, be the weights for the $(p+1)$-point Gauss-Lobatto quadrature. Then for $k=2, \ldots, p$,

$$
\left(w_{p}^{\prime}, \phi_{k}^{\prime}\right)=-\left(w_{p}^{\prime \prime}, \phi_{k}\right)=-\sum_{j=0}^{p} w_{p}^{\prime \prime}\left(x_{j}\right) \phi_{k}\left(x_{j}\right) \omega_{j}=\sum_{j=0}^{p} f\left(x_{j}\right) \phi_{k}\left(x_{j}\right) \omega_{j}=\left(f, \phi_{k}\right)_{*} .
$$

Note that the $(p+1)$-point Gauss-Lobatto quadrature rule $\left(f, \phi_{j}\right)_{*}$ is exact for polynomials of degrees up to $2 p-1$. Therefore, the solution $w_{p}$ can be expressed by

$$
w_{p}(x)=\sum_{j=2}^{p} c_{j-1} \phi_{j}(x), \quad c_{j-1}=\frac{2 j-1}{2}\left(f, \phi_{j}\right)_{*} .
$$

We want to estimate the error between $u_{p}$ and $w_{p}$. This error is the influence of numerical interpolation and integration. Towards this end, we introduce an auxiliary problem: Find $v_{p} \in \mathcal{P}_{p} \cap H_{0}^{1}[-1,1]$ such that

$$
\left(v_{p}^{\prime}, \phi_{j}^{\prime}\right)=\left(f_{p}, \phi_{j}\right), \quad j=2, \ldots, p,
$$

where $f_{p}$ is the Lagrange interpolation of $f$ at $p+1$ Gauss-Lobatto points. Since the quadrature rule $(\cdot, \cdot)_{*}$ uses only the function values at the Gauss-Lobatto points where $f_{p}$ is interpolated, then

$$
\left(f, \phi_{j}\right)_{*}=\left(f_{p}, \phi_{j}\right)_{*}, \quad j=2, \ldots, p .
$$

Recall that the quadrature rule $(\cdot, \cdot)_{*}$ is exact for polynomials of degree $\leq 2 p-1$. From this it is clear that

$$
\left(f_{p}, \phi_{j}\right)_{*}=\left(f_{p}, \phi_{j}\right), \quad j=2, \ldots, p-1 .
$$

We see that $w_{p}$ and $v_{p}$ differ only at the last coefficient. If we denote

$$
\bar{c}_{p-1}=\frac{2 p-1}{2}\left(f_{p}, \phi_{p}\right)
$$

then

$$
v_{p}(x)-w_{p}(x)=\frac{2 p-1}{2}\left(\left(f_{p}, \phi_{p}\right)-\left(f_{p}, \phi_{p}\right)_{*}\right) \phi_{p}(x) .
$$

Theorem 3.1. Let $w_{p}$ and $v_{p}$ be solutions of (3.1) and (3.2), respectively. Then

$$
\left(v_{p}-w_{p}\right)(x)=-\frac{(p+1) 2^{p} p !}{p(2 p+1)(2 p) !} f^{(p)}\left(\xi_{p}\right) \phi_{p}(x), \quad \xi_{p} \in(-1,1) .
$$

Proof. The numerical integration error is given by [7, p. 104, (2.7.1.13)]

$$
\left(f_{p}, \phi_{p}\right)-\left(f_{p}, \phi_{p}\right)_{*}=-\frac{(p+1) 2^{2 p+1}(p !)^{4}}{p(2 p+1)[(2 p) !]^{3}}\left(f_{p} \phi_{p}\right)^{(2 p)} .
$$

By the Newton-Leibnitz formula

$$
\begin{aligned}
\left(f_{p} \phi_{p}\right)^{(2 p)} & =\left(\begin{array}{c}
2 p \\
p
\end{array}\right) f_{p}^{(p)} \phi_{p}^{(p)} \\
& =\left(\begin{array}{c}
2 p \\
p
\end{array}\right)\left(\begin{array}{c}
2 p-2 \\
p-1
\end{array}\right) \frac{(p-1) !}{2^{p-1}} f_{p}^{(p)} \\
& =\frac{[(2 p) !]^{2}}{(2 p-1)(p !)^{3} 2^{p}} f^{(p)}\left(\xi_{p}\right) .
\end{aligned}
$$


Here we have used the fact that

$$
\phi_{p}^{(p)}(x)=L_{p-1}^{(p-1)}(x)=\left(\begin{array}{c}
2 p-2 \\
p-1
\end{array}\right) \frac{(p-1) !}{2^{p-1}} .
$$

Note that $f_{p}^{(p)}=f\left[x_{0}, x_{1}, \ldots, x_{p}\right] p$ !. Substituting (3.6) into (3.5) yields

$$
\left(f_{p}, \phi_{p}\right)-\left(f_{p}, \phi_{p}\right)_{*}=-\frac{(p+1) 2^{p+1} p !}{p\left(4 p^{2}-1\right)(2 p) !} f^{(p)}\left(\xi_{p}\right) .
$$

The conclusion follows from (3.3).

Applying Stirling's formula and condition (M) (note that $f^{(p)}=-u^{(p+2)}$ ) to Theorem 3.1, it is straightforward to obtain estimates for $v_{p}-w_{p}$ in any norm, especially the following.

\section{Corollary 3.1.}

$$
\begin{aligned}
& \left\|v_{p}-w_{p}\right\|_{L^{2}[-1,1]}=O\left[\frac{1}{\sqrt{p}}\left(\frac{e M}{2 p}\right)^{p+2}\right], \quad\left\|v_{p}^{\prime}-w_{p}^{\prime}\right\|_{L^{2}[-1,1]}=O\left[\sqrt{p}\left(\frac{e M}{2 p}\right)^{p+2}\right], \\
& \text { 3.9) }\left\|v_{p}-w_{p}\right\|_{L^{\infty}[-1,1]}=O\left(\frac{e M}{2 p}\right)^{p+2}, \quad\left\|v_{p}^{\prime}-w_{p}^{\prime}\right\|_{L^{\infty}[-1,1]}=O\left[p\left(\frac{e M}{2 p}\right)^{p+2}\right] .
\end{aligned}
$$

Theorem 3.2. Let $u_{p}$ and $v_{p}$ be solutions of (2.4) and (3.2), respectively. Assume that $u$ satisfies condition $(\mathrm{M})$. Then

$$
\begin{gathered}
\left\|u_{p}-v_{p}\right\|_{L^{2}[-1,1]} \leq \frac{2}{\pi}\left\|u_{p}^{\prime}-v_{p}^{\prime}\right\|_{L^{2}[-1,1]} \leq \frac{C}{\sqrt{p}}\left(\frac{e M}{2 p}\right)^{p+2} \\
\left\|u_{p}-v_{p}\right\|_{L^{\infty}[-1,1]} \leq \frac{C}{\sqrt{p}}\left(\frac{e M}{2 p}\right)^{p+2}, \quad\left\|u_{p}^{\prime}-v_{p}^{\prime}\right\|_{L^{\infty}[-1,1]} \leq C\left(\frac{e M}{2 p}\right)^{p+2} .
\end{gathered}
$$

Proof. By (2.4) and (3.2), the error expression can be decomposed into two parts

$$
\left(u_{p}-v_{p}\right)(x)=\sum_{j=2}^{p} \frac{2 j-1}{2}\left(f-f_{p}, \phi_{j}\right) \phi_{j}(x)=R_{p}(x)+r_{p}(x),
$$

where by (1.2)

$$
\begin{aligned}
& R_{p}(x)=c_{1}(p) \sum_{j=2}^{p} \frac{2 j-1}{2}\left(\left(f\left[x_{0}, \ldots, x_{p}, \cdot\right]-f\left[x_{0}, \ldots, x_{p}, 0\right]\right)\left(L_{p+1}-L_{p-1}\right), \phi_{j}\right) \phi_{j}(x), \\
& r_{p}(x)=\frac{2 p-3}{2} c_{1}(p) f\left[x_{0}, \ldots, x_{p}, 0\right]\left(L_{p+1}-L_{p-1}, \phi_{p-1}\right) \phi_{p-1}(x) \\
& (3.13) \quad=-c_{1}(p) \frac{f^{(p+1)}\left(\eta_{p}\right)}{(p+1) !} \frac{1}{2 p-1} \phi_{p-1}(x) .
\end{aligned}
$$

Here we have used the fact that

$$
\left(L_{p+1}-L_{p-1}, \phi_{j}\right)=0, \quad j \neq p-1, \quad\left(L_{p+1}-L_{p-1}, \phi_{p-1}\right)=-\frac{2}{(2 p-1)(2 p-3)} .
$$


The expressions for $u_{p}^{\prime}-v_{p}^{\prime}$ as well as $R_{p}^{\prime}$ and $r_{p}^{\prime}$ can be obtained by replacing $\phi_{j}(x)$ with $L_{j-1}(x)$. Observe that

$$
\begin{aligned}
& \left|\left(\left(f\left[x_{0}, \ldots, x_{p}, \cdot\right]-f\left[x_{0}, \ldots, x_{p}, 0\right]\right)\left(L_{p+1}-L_{p-1}\right), \phi_{j}\right)\right| \\
& \quad \leq \frac{\left\|f^{(p+2)}\right\|_{L^{\infty}[-1,1]}}{(p+2) !}\left\|L_{p+1}-L_{p-1}\right\|_{L^{2}[-1,1]}\left\|\phi_{j}\right\|_{L^{2}[-1,1]},
\end{aligned}
$$

and $\left\|L_{j}\right\|_{L^{\infty}[-1,1]}=1$. We have

$$
\begin{aligned}
& \left\|R_{p}^{\prime}\right\|_{L^{\infty}[-1,1]} \\
& \quad \leq c_{1}(p) \frac{\left\|f^{(p+2)}\right\|_{L^{\infty}[-1,1]}}{(p+2) !}\left\|L_{p+1}-L_{p-1}\right\|_{L^{2}[-1,1]} \sum_{j=2}^{p} \frac{2 j-1}{2}\left\|\phi_{j}\right\|_{L^{2}[-1,1]} \\
& \quad \leq 2 c_{1}(p) \frac{C M^{p+2}}{(p+2) !} .
\end{aligned}
$$

Here we have used (1.8) and the fact that

$$
\sum_{j=2}^{p} \frac{2 j-1}{2}\left\|\phi_{j}\right\|_{L^{2}[-1,1]}=\sum_{j=2}^{p} \sqrt{\frac{2 j-1}{(2 j-3)(2 j+1)}} \leq \int_{1}^{p} \frac{d x}{\sqrt{2 x-1}}=\sqrt{2 p-1}-1 .
$$

Similarly, note that $\left\|\phi_{j}\right\|_{L^{\infty}[-1,1]} \leq 2 /(2 j-1)$. We have

$$
\begin{aligned}
& \left\|R_{p}\right\|_{L^{\infty}[-1,1]} \\
& \quad \leq c_{1}(p) \frac{\left\|f^{(p+2)}\right\|_{L^{\infty}[-1,1]}}{(p+2) !}\left\|L_{p+1}-L_{p-1}\right\|_{L^{2}[-1,1]} \sum_{j=2}^{p}\left\|\phi_{j}\right\|_{L^{2}[-1,1]} \\
& \quad<2 c_{1}(p) \frac{C M^{p+2}}{(p+2) !}\left\|L_{p+1}-L_{p-1}\right\|_{L^{2}[-1,1]} .
\end{aligned}
$$

Here we have used the fact that

$$
\sum_{j=2}^{p}\left\|\phi_{j}\right\|_{L^{2}[-1,1]}=2 \sum_{j=2}^{p} \frac{1}{\sqrt{(2 j-3)(2 j-1)(2 j+1)}}<2 \int_{1}^{p} \frac{d x}{(2 x-1)^{3 / 2}}<2 .
$$

Apply (1.3), condition (M), and Stirling's formula to (3.13), (3.14), and (3.15), we obtain the estimates (3.11). Similarly, we obtain the estimate

$$
\begin{aligned}
\left\|R_{p}^{\prime}\right\|_{L^{2}[-1,1]}^{2} & \\
\leq & c_{1}(p)^{2} \frac{\left\|f^{(p+2)}\right\|_{L^{\infty}[-1,1]}^{2}}{(p+2) !^{2}}\left\|L_{p+1}-L_{p-1}\right\|_{L^{2}[-1,1]}^{2} \\
& \times \sum_{j=2}^{p} \frac{(2 j-1)^{2}}{4}\left\|\phi_{j}\right\|_{L^{2}[-1,1]}^{2}\left\|L_{j-1}\right\|_{L^{2}[-1,1]}^{2} \\
\leq & \frac{8(2 p+1)}{3(2 p+3)(2 p-1)} c_{1}(p)^{2} \frac{C^{2} M^{2(p+2)}}{(p+2) !^{2}}
\end{aligned}
$$

by using (1.8), (1.9), and condition (M). The estimate for $\left\|r_{p}^{\prime}\right\|_{L^{2}[-1,1]}$ can be obtained similarly by replacing $\phi_{p-1}$ with $L_{p-2}$ in (3.13). The semi- $H^{1}$ norm estimate follows by recalling (1.3) and Stirling's formula.

The $L^{2}$-error is a direct application of the Poincare inequality. 
Superconvergence. We are interested in the error between $u$ and $w_{p}$. Consider the decomposition

$$
\left(u-w_{p}\right)(x)=\left(u-u_{p}\right)(x)+\left(u_{p}-v_{p}\right)(x)+\left(v_{p}-w_{p}\right)(x) .
$$

Corollary 2.1, Theorem 3.2, and Corollary 3.1 provide error bounds for all three terms on the right-hand side, respectively. We see that the latter two terms converge faster than the first term by at least a factor of $p$. Therefore, the error $u-w_{p}$ is majorized by $u-u_{p}$.

In order to investigate the superconvergence property, we denote by $g_{j}, j=$ $1, \ldots, p$, the Gaussian points, and we let $\|\cdot\|_{G}$ be the Gauss quadrature with these $p$ Gaussian points. We also recall that the $x_{j}$ are the Gauss-Lobatto points and that $\|\cdot\|_{*}$ is the $(p+1)$-point Gauss-Lobatto quadrature.

Theorem 3.3. Let $u$ and $w_{p}$ be solutions of (2.2) and (3.1), respectively. Assume that $u$ satisfies condition $(\mathrm{M})$ and the nondegenerate condition $b_{p} \neq 0$. Then when $(2 p+1)(2 p+3)>2 M^{2}$, we have

$$
\begin{aligned}
\max _{1 \leq j \leq p-1}\left|\left(u-w_{p}\right)\left(x_{j}\right)\right| & \leq C\left(\frac{e M}{2 p}\right)^{p+2}, \\
\max _{1 \leq j \leq p}\left|\left(u-w_{p}\right)^{\prime}\left(g_{j}\right)\right| & \leq C p\left(\frac{e M}{2 p}\right)^{p+2}, \\
\left\|u-w_{p}\right\|_{*} & \leq \frac{C}{\sqrt{p}}\left(\frac{e M}{2 p}\right)^{p+2}, \\
\left\|u^{\prime}-w_{p}^{\prime}\right\|_{G} & \leq C \sqrt{p}\left(\frac{e M}{2 p}\right)^{p+2} .
\end{aligned}
$$

Proof. By the decomposition (3.16), Theorem 3.2, and Corollary 3.1, we have

$$
\begin{aligned}
\left|\left(u-w_{p}\right)\left(x_{j}\right)\right| & \leq\left|\left(u-u_{p}\right)\left(x_{j}\right)\right|+C\left(\frac{e M}{2 p}\right)^{p+2} \\
& \approx\left|b_{p+1} \phi_{p+2}\left(x_{j}\right)\right|+C\left(\frac{e M}{2 p}\right)^{p+2} \leq C\left(\frac{e M}{2 p}\right)^{p+2} .
\end{aligned}
$$

Note that $\phi_{p+1}\left(x_{j}\right)=0$ and therefore the dominate term in $\left(u-u_{p}\right)\left(x_{j}\right)$ is $b_{p+1} \phi_{p+2}\left(x_{j}\right)$. This proves (3.17). Similarly,

$$
\begin{aligned}
\left|\left(u-w_{p}\right)^{\prime}\left(g_{j}\right)\right| & =\left|\left(u-u_{p}\right)^{\prime}\left(g_{j}\right)\right|+C\left(\frac{e M}{2 p}\right)^{p+1} \\
& \approx\left|b_{p+1} L_{p+1}\left(g_{j}\right)\right|+C\left(\frac{e M}{2 p}\right)^{p+1} \leq C\left(\frac{e M}{2 p}\right)^{p+1} .
\end{aligned}
$$

Here we have used the fact that $L_{p}\left(g_{j}\right)=0$. This establishes (3.18). Next,

$$
\begin{aligned}
\left\|u^{\prime}-w_{p}^{\prime}\right\|_{*} & \approx\left\|u^{\prime}-u_{p}^{\prime}\right\|_{*} \approx\left\|b_{p+1} L_{p+1}\right\|_{*} \approx\left\|b_{p+1} L_{p+1}\right\| \\
& =\frac{2\left|b_{p+1}\right|}{2 p+3} \leq \frac{\sqrt{2} c}{\sqrt{\pi p}}\left(\frac{e M}{2(p+1)}\right)^{p+1},
\end{aligned}
$$

which is equivalent to (3.20). The estimate of (3.19) is similar. 
Theorem 3.4. Assume the same hypotheses as in Theorem 3.3. Let $u$ be even (odd) and let $p$ be odd (even). We have

$$
\begin{aligned}
\max _{1 \leq j \leq p}\left|\left(u-w_{p}\right)^{\prime}\left(g_{j}\right)\right| & \leq C\left(\frac{e M}{2 p}\right)^{p+2}, \\
\left\|u^{\prime}-w_{p}^{\prime}\right\|_{G} & \leq \frac{C}{\sqrt{p}}\left(\frac{e M}{2 p}\right)^{p+2} .
\end{aligned}
$$

Proof. With the even-odd assumption, $f_{p} \phi_{p}$ is an odd function and therefore

$$
\left(f_{p}, \phi_{p}\right)=0, \quad\left(f_{p}, \phi_{p}\right)_{*}=0 .
$$

As a consequence, $v_{p}-w_{p}=0$ in the decomposition (3.16). Hence by Theorem 3.2, we have

$$
\begin{aligned}
\left|\left(u-w_{p}\right)^{\prime}\left(g_{j}\right)\right| & \leq\left|\left(u-u_{p}\right)^{\prime}\left(g_{j}\right)\right|+C\left(\frac{e M}{2 p}\right)^{p+2} \\
& \approx\left|b_{p+2} L_{p+2}\left(g_{j}\right)\right|+C\left(\frac{e M}{2 p}\right)^{p+2} \leq C\left(\frac{e M}{2 p}\right)^{p+2} .
\end{aligned}
$$

Here we have used the fact that $L_{p}\left(g_{j}\right)=0$ and $b_{p+1}=0$. This establishes (3.21). Next,

$$
\begin{aligned}
\left\|u^{\prime}-w_{p}^{\prime}\right\|_{*} & \approx\left\|u^{\prime}-u_{p}^{\prime}\right\|_{*} \approx\left\|b_{p+2} L_{p+2}\right\|_{*} \approx\left\|b_{p+2} L_{p+2}\right\| \\
& =\frac{2\left|b_{p+2}\right|}{2 p+5} \leq \frac{\sqrt{2} c}{\sqrt{\pi p}}\left(\frac{e M}{2(p+2)}\right)^{p+2},
\end{aligned}
$$

which is equivalent to (3.22).

Remark. As we mentioned earlier, the collocation solution $w_{p}$ is equivalent to applying the $(p+1)$-point Gauss-Lobatto quadrature to obtain $\left(f, \phi_{j}\right)_{*}$. However, the same error bounds hold for any $p$-point or $(p+1)$-point quadrature rule when constructed by orthogonal polynomials and is exact for polynomials of degree $2 p-1$. Again, this is confirmed by numerical tests. We have actually tested $p$-point Gauss quadrature, $p$-point Chebyshev quadrature, and $(p+1)$-point Chebyshev-Lobatto quadrature. They show no essential difference in delivering the numerical results.

General cases. Similar results can be developed for general two-point boundary value problems of type

$$
-\left(a u^{\prime}\right)^{\prime}+b u=f, \quad u( \pm 1)=0, \quad a(x) \geq \alpha>0, \quad b(x) \geq 0,
$$

or in its weak form: Find $u \in H_{0}^{1}[-1,1]$ such that

$$
A(u, v)=\left(a u^{\prime}, v^{\prime}\right)+(b u, v)=(f, v), \quad \forall v \in H_{0}^{1}[-1,1] .
$$

In fact, let $u_{p}$ be the Galerkin spectral approximation in the finite dimensional polynomial space of degree $p$. Then we have

$$
\left\|u-u_{p}\right\|_{A} \leq\left\|u-\pi_{p} u\right\|_{A},
$$

where $\|\cdot\|_{A}$ is the energy norm induced by the bilinear form and $\pi_{p}$ is the projection operator such that $\left(\pi_{p} u\right)^{\prime}$ is the truncated Legendre expansion of $u^{\prime}$ defined in Section 2. In this way, all analysis in Section 2 can be applied here. As for the spectral collocation, some more numerical integration error estimates are needed. 


\section{Numerical tests}

We consider only a simple model problem:

$$
u^{\prime \prime}+b u=f, \quad u( \pm 1)=0 .
$$

The Legendre collocation method is to find $\vec{w}_{p}$ such that $\left(D_{p}^{2}+b I\right) \vec{w}_{p}=\vec{f}$ with $[\underline{6}$, p. 65]

$$
D_{p}(j, j)=0, \quad D_{p}(i, j)=\frac{L_{p}\left(x_{i}\right)}{L_{p}\left(x_{j}\right)} \frac{1}{x_{i}-x_{j}}, \quad i \neq j, \quad \vec{f}=\left(\begin{array}{c}
f\left(x_{1}\right) \\
\vdots \\
f\left(x_{p-1}\right)
\end{array}\right),
$$

where $x_{1}<x_{2}<\cdots<x_{p-1}$ are interior Lobatto points, zeros of $L_{p}^{\prime}$. The solution $\vec{w}_{p}$ provides an approximation at the Lobatto points. In order to obtain derivative values, we define

$$
w_{p}(x)=\sum_{k=1}^{p-1} c_{k} \psi_{k+1}(x), \quad \psi_{k+1}(x)=\left(1-x^{2}\right) L_{p}^{\prime}(x) .
$$

Then

$$
w_{p}^{\prime}(x)=-\sum_{k=1}^{p-1} c_{k} k(k+1) L_{k}(x),
$$

where $\vec{c}$ can be obtained from $T \vec{c}=\vec{w}_{p}$ with

$$
T=\left(\begin{array}{cccc}
\psi_{2}\left(x_{1}\right) & \psi_{3}\left(x_{1}\right) & \cdots & \psi_{p}\left(x_{1}\right) \\
\psi_{2}\left(x_{2}\right) & \psi_{3}\left(x_{2}\right) & \cdots & \psi_{p}\left(x_{2}\right) \\
\vdots & \vdots & \ddots & \vdots \\
\psi_{2}\left(x_{p-1}\right) & \psi_{3}\left(x_{p-1}\right) & \cdots & \psi_{p}\left(x_{p-1}\right)
\end{array}\right)
$$

Fortunately, $T$ has an explicit inverse [12]:

$$
T^{-1}=\frac{2 p}{p+1}\left(\begin{array}{cccc}
\frac{1-x_{1}^{2}}{\psi_{p}^{2}\left(x_{1}\right)} \frac{\psi_{2}\left(x_{1}\right)}{s_{2}} & \frac{1-x_{2}^{2}}{\psi_{p}^{2}\left(x_{2}\right)} \frac{\psi_{2}\left(x_{2}\right)}{s_{2}} & \cdots & \frac{1-x_{p-1}^{2}}{\psi_{p}^{2}\left(x_{p-1}\right)} \frac{\psi_{2}\left(x_{p-1}\right)}{s_{2}} \\
\frac{1-x_{1}^{2}}{\psi_{p}^{2}\left(x_{1}\right)} \frac{\psi_{3}\left(x_{1}\right)}{s_{3}} & \frac{1-x_{2}^{2}}{\psi_{p}^{2}\left(x_{2}\right)} \frac{\psi_{3}\left(x_{2}\right)}{s_{3}} & \cdots & \frac{1-x_{p-1}^{2}}{\psi_{p}^{2}\left(x_{p-1}\right)} \frac{\psi_{3}\left(x_{p-1}\right)}{s_{3}} \\
\vdots & \vdots & \ddots & \vdots \\
\frac{1-x_{1}^{2}}{\psi_{p}^{2}\left(x_{1}\right)} \frac{\psi_{p}\left(x_{1}\right)}{s_{p}} & \frac{1-x_{2}^{2}}{\psi_{p}^{2}\left(x_{2}\right)} \frac{\psi_{p}\left(x_{2}\right)}{s_{p}} & \cdots & \frac{1-x_{p-1}^{2}}{\psi_{p}^{2}\left(x_{p-1}\right)} \frac{\psi_{p}\left(x_{p-1}\right)}{s_{p}}
\end{array}\right),
$$

with $s_{k}=\frac{2 k(k-1)}{2 k-1}$. Note that $\psi_{k+1}^{\prime}(x)=-k(k+1) L_{k}(x)$.

We choose $b=10$ and $u=\sin 4 \pi x$. Therefore, $M=4 \pi$. The asymptotic condition $(2 p+1)(2 p+3)>2 M^{2}$ in Theorem 3.3 is about $\sqrt{2}(p+1)>M$, which suggests $p>8$. Indeed, for smaller $p$, we observe some oscillatory behavior in the error.

Figure 1depicts the error (the $y$-axis) $\max _{1 \leq j \leq p-1}\left|\left(u-w_{p}\right)\left(x_{j}\right)\right|$ against $\left(\frac{e M}{2 p}\right)^{p+2}$ for $p=20,21, \ldots, 30$. We observe straight lines for both even (o) and odd $(*)$ $p$. To see different rates for odd and even $p$, Figure 3 plots the ratio $\left(\frac{e M}{2 p}\right)^{p+2}$ : $\sqrt{p} \max _{1 \leq j \leq p}\left|\left(u-w_{p}\right)\left(x_{j}\right)\right|$. This ratio is a constant for odd $p$ (comparing with (3.17) 


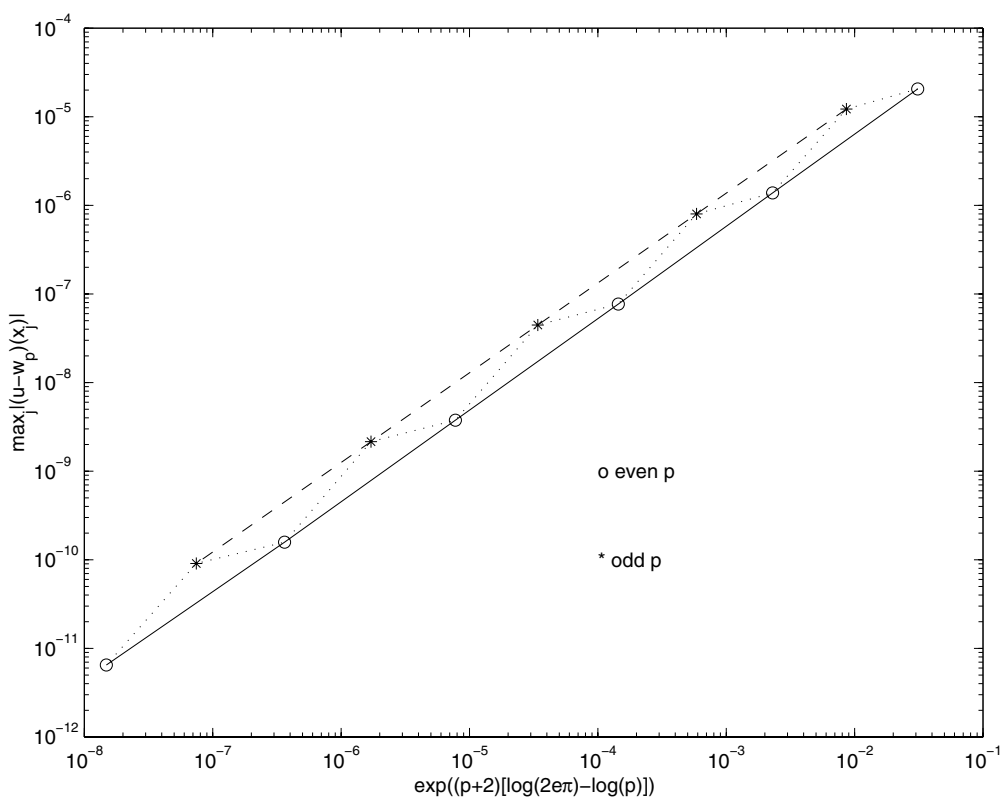

FigURE 1. Lobatto points convergent rate

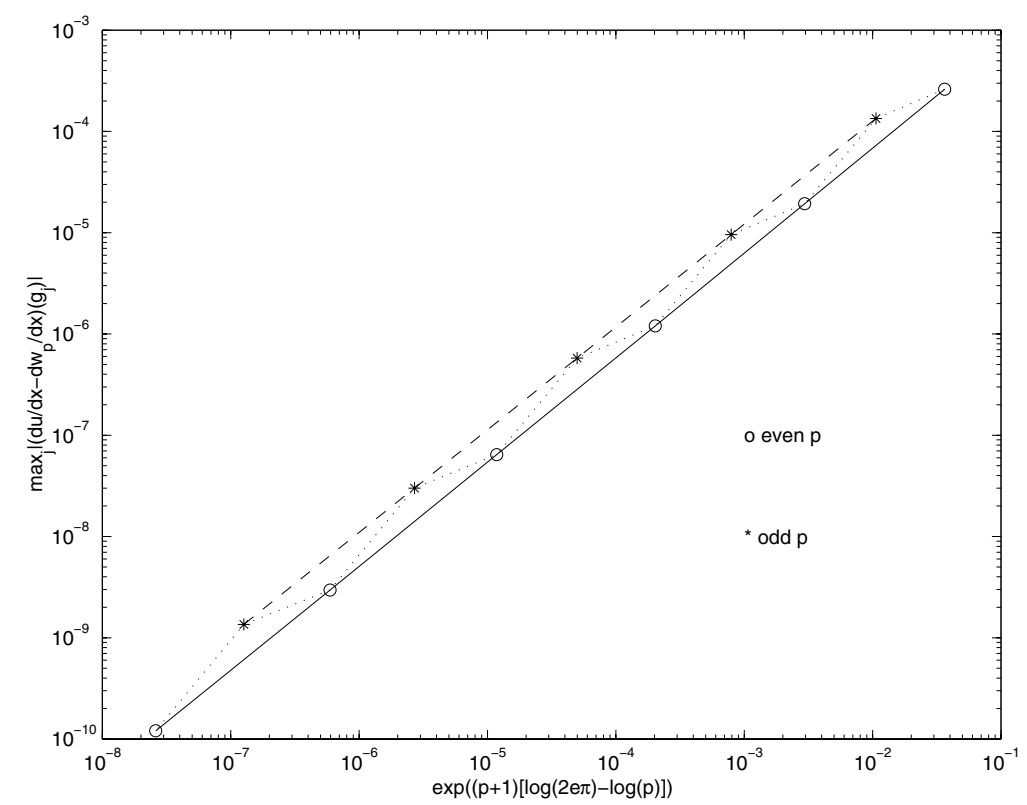

FIGURE 2. Gaussian points convergence rate

and (3.19) $)$ and increases linearly for even $p$, indicating an order-two superconvergence rate. 


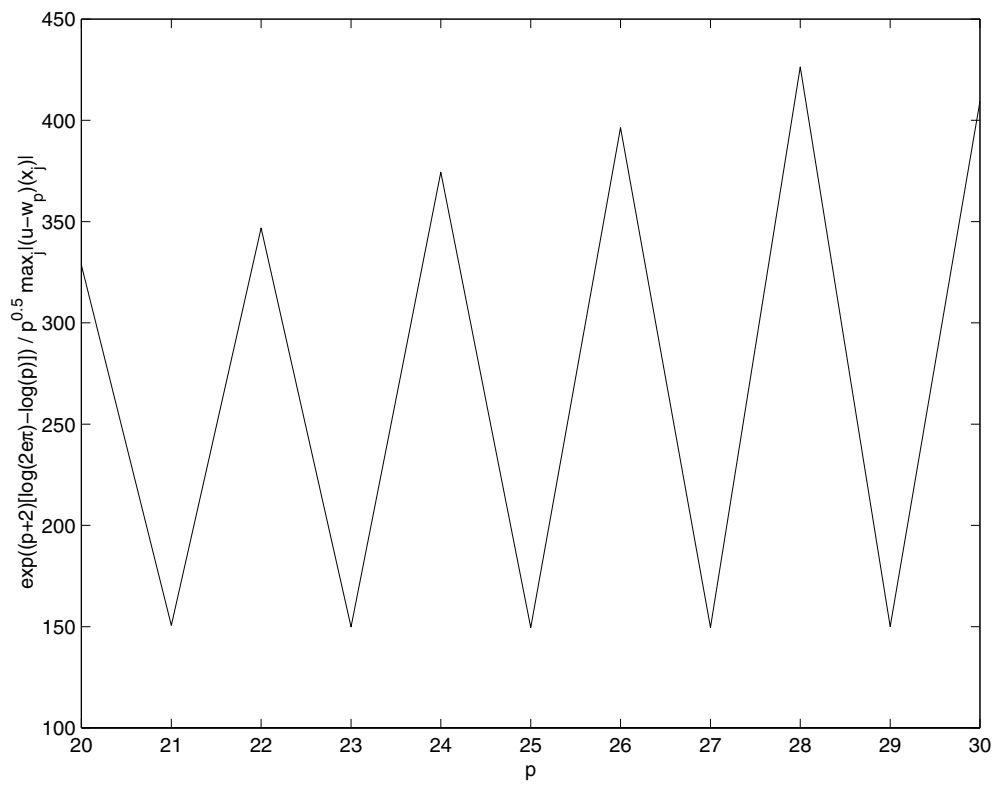

Figure 3. Lobatto points convergent rate

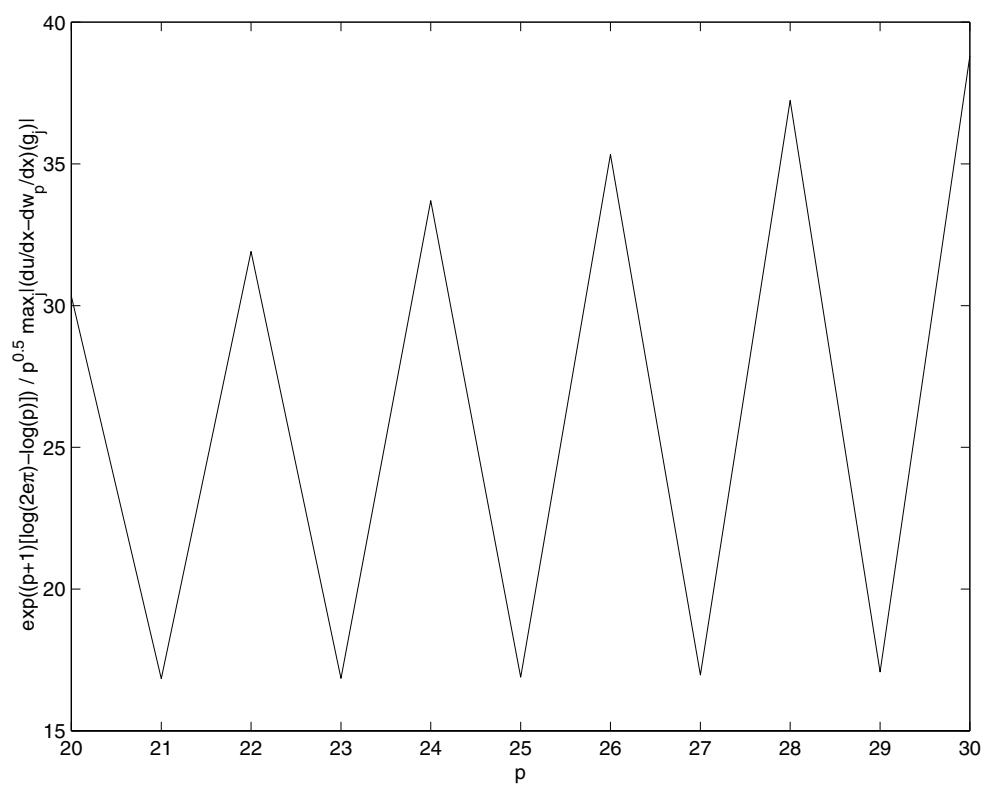

FiguRE 4. Gaussian points convergent rate

Figure 2 depicts the error (the $y$-axis) $\max _{1 \leq j \leq p}\left|\left(u^{\prime}-w_{p}^{\prime}\right)\left(g_{j}\right)\right|$ against $\left(\frac{e M}{2 p}\right)^{p+1}$, and Figure 4 plots the ratio $\left(\frac{e M}{2 p}\right)^{p+1}: \sqrt{p} \max _{1 \leq j \leq p}\left|\left(u^{\prime}-w_{p}^{\prime}\right)\left(g_{j}\right)\right|$. Again we observe 
superconvergence of order-one for odd $p$ (comparing with (3.18) and (3.20) ) and order-two for even $p$ (comparing with (3.21) and (3.22)).

\section{FinAl REMARKS}

Comparing with Theorem 2.1, there is a completely different strategy for proving the supergeometric rate of convergence for the usual Fourier and Chebyshev expansions; see, e.g., [11, [8, p. 37], and [4]. The asymptotics of the Legendre polynomials are messier than Fourier or Chebyshev, making the task much harder here.

The Legendre polynomials have been used as basis functions in the $p$-version finite element community since late 1970s; see [19] and references therein. In the early 1990s, Shen introduced them to the spectral method community combined with fast solvers [17, which made the method more appealing.

The counterpart error bound for the $h p$-version finite element is in the form $\left(\frac{e M h}{2 p}\right)^{p+\alpha}$, where $h$ is the mesh size and $\alpha=1,2$.

Entire functions and condition (M). Consider entire function

$$
f(z)=\sum_{n=0}^{\infty} c_{n} z^{n}, \quad \lim _{n \rightarrow \infty} \sqrt[n]{\left|c_{n}\right|}=0 .
$$

A spectral approximation of $f$ converges at a certain rate depending on the way $c_{n}$ decreases. In order to see the relativeness of condition (M), we need some notation. Here we follow [10]. Define

$$
M_{f}(r)=\max _{|z|=r}|f(z)| .
$$

If $M_{f}(r)$ has a "minimum" upper-bound of type $e^{\sigma r^{\rho}}$, then we say that the entire function $f$ is of order $\rho$ and of type $\sigma$. To be more precise,

$$
\rho=\lim _{n \rightarrow \infty} \sup \frac{\log \log M_{f}(r)}{\log r}, \quad \sigma=\lim _{r \rightarrow \infty} \sup \frac{\log M_{f}(r)}{r^{\rho}} .
$$

Indeed, $\rho$ and $\sigma$ are determined by $c_{n}$ in the following way:

$$
\rho=\lim _{r \rightarrow \infty} \sup \frac{n \log n}{-\log \left|c_{n}\right|}, \quad \sigma=\frac{1}{\rho e} \lim _{n \rightarrow \infty} \sup n \sqrt[n]{\left|c_{n}\right|^{\rho}} .
$$

When $\rho=1$, the entire function $f$ is called the exponential type. One of the most significant properties of this kind of function was due to Bernstein [10, p. 227]: If $f(z)$ is an entire function of exponential type $\sigma>0$ and $|f(x)| \leq C$ for $-\infty<x<$ $\infty$, then $\left|f^{\prime}(x)\right| \leq C \sigma$ for $-\infty<x<\infty$. The equality sign is attained here for some $x$ if and only if $f(z)=c_{1} \cos \sigma z+c_{2} \sin \sigma z$.

By this property, entire functions of exponential type bounded on the real line satisfy condition (M) with $\sigma=M$. Then all results in the paper are valid.

As for the general case when $\rho \neq 1$, the rate of convergence is of the type (comparing with Theorem 2.1):

$$
\left(\frac{e M \rho}{p}\right)^{\frac{p}{\rho}+1} 2^{-p}, \quad \sigma=M .
$$


Other cases are

(1) $0<\rho<\infty$ : $f$ is of order $\rho$ and of maximum (minimum) type if $\sigma=\infty$ $(\sigma=0)$.

(2) $\rho=\infty: f$ is of infinite order.

(3) $\rho=0: f$ is of zero order.

Spectral approximations of different types of entire functions have different convergence rates. Finally, it is worthy to point out that the results in the paper cover more than entire functions, since condition $(\mathrm{M})$ is required only on a finite interval (the solution domain), not the whole real line or the whole complex plain.

\section{APPENDIX}

Stirling's formula is

$$
\sqrt{2 \pi} n^{n+1 / 2}<n ! e^{n}<\sqrt{2 \pi} n^{n+1 / 2}\left(1+\frac{1}{4 n}\right) .
$$

A rough approximation was given by Stirling in 1730:

$$
n ! \approx\left(\frac{n}{e}\right)^{n} \sqrt{2 \pi n}
$$

The relative error is less than $1 \%$ from $n \geq 9$ and less than $0.1 \%$ from $n \geq 84$. A more accurate approximation is [1, (4.48)]

$$
n ! \approx\left(\frac{n}{e}\right)^{n} \sqrt{2 \pi\left(n+\frac{1}{6}\right)}
$$

The relative error is less than $1 \%$ from $n \geq 3$ and less than $0.1 \%$ from $n \geq 9$. Another useful result is

$$
(2 n-1) ! ! \approx \frac{\sqrt{(2 n) !}}{\sqrt[4]{\pi\left(n+\frac{1}{4}\right)}}
$$

which comes from [2],

$$
\pi=\lim _{n \rightarrow \infty}\left(\frac{2^{2 n}}{\left(\begin{array}{c}
2 n \\
n
\end{array}\right)}\right)^{2}\left(n+\frac{1}{4}\right)^{-1} .
$$

\section{ACKNOWLEDGMENTS}

I would like to thank Professor J. Shen at Purdue University for bringing this topic to my attention and for his helpful discussions. Thanks also go to the referee who made many constructive suggestions that improved the presentation.

\section{REFERENCES}

[1] J. Arndt and C. Haenel, $\pi$-Unleashed, Springer, Berlin, 2001. MR.1842703 (2002g:11185)

[2] F.L. Bauer, Decrypted Secrets, Methods and Maxims of Cryptology, 2nd, revised, and extended edition, Springer-Verlag, Heidelberg, 2000. MR1762414 (2001a:94021)

[3] C. Bernardi and Y. Maday, Spectral Methods. In Handbook of Numerical Analysis, Vol. V, P.G. Ciarlet and J.-L. Lions eds., North-Holland (1997), 209-485. MR.1470226

[4] J.P. Boyd, The rate of convergence of Fourier coefficients for entire functions of infinite order with application to the Weideman-Cloot-Sinh-Mapping for pseudospectral computations on an infinite interval, J. Comp. Phys. 110 (1994), 360-372. MR 1267887

[5] J.P. Boyd, Chebyshev and Fourier Spectral Methods, 2nd edition, Dover, New York, 2001. MR.1874071 (2002k:65160) 
[6] C. Canuto, M.Y. Hussaini, A. Quarteroni, and T.A. Zang, Spectral Methods in Fluid Dynamics, Springer-Verlag, New York, 1988. MR0917480 (89m:76004)

[7] P.J. Davis and P. Rabinowitz, Methods of Numerical Integration, 2nd ed., Academic Press, Boston, 1984. MR.0760629 (86d:65004)

[8] D. Gottlieb and T.A. Orszag, Numerical Analysis of Spectral Methods: Theory and Applications, SIAM, Philadelphia, 1977. MR0520152 (58:24983)

[9] G.E. Karniadakis and S.J. Sherwin, Spectral/hp Element Methods for CFD, Oxford University Press, New York, 1999. MR1696933(2000h:76120)

[10] B.Ya. Levin, Lectures on Entire Functions, Translations of Mathematical Monographs, Vol. 150, AMS, Providence, 1996. MR1400006 (97j:30001)

[11] Y.L. Luke, The Special Functions and Their Applications, Vol. 1, Academic Press, New York, 1969. MR0241700 (39:3039)

[12] P.K. Moore, Applications of Lobatto polynomials to an adaptive finite element method: A posteriori error estimates for hp-adaptivity and grid-to-grid interpolation, Numer. Math. 94-2 (2003), 367-401. MR1974560 (2004e:65105)

[13] R. Peyret, Specrtral Methods for Incompressible Viscous Flow, Springer, New York, 2002. MR.1897544 (2003d:65002)

[14] G.M. Phillips, Interpolation and Approximation by Polynomials, Springer, New York, 2003. MR.1975918 (2004c:41001)

[15] G. Sansone, Orthogonal Functions, Dover, New York, 1991. MR1118381 (92e:42015)

[16] C. Schwab, $p$ - and hp-Finite Element Methods, Oxford University Press, 1998. MR1695813 (2000d:65003)

[17] J. Shen, Effective spectral-Galerkin method I. Direct solvers for the second and fourth order equations using Legendre polynomials, SIAM J. Sci. Comput. 15-6 (1994), 1489-1505. MR:1298626(95j:65150)

[18] J. Shen, A new dual-Petrov-Galerkin method for third and higher odd-order differential equations: Application to the KDV equation, SIAM J. Numer. Anal. 41-5 (2003), 15951619. MR 2034999

[19] B. Szabó and I. Babuška, Finite Element Analysis, John Wiley \& Sons, New York, 1991. MR1164869 (93f:73001)

[20] G. Szegö, Orthogonal Polynomials, 4th edition, AMS Colloq. Public, Vol. 23, 1975. MR0372517 (51:8724)

[21] L.N. Trefethen, Spectral Methods in Matlab, SIAM, 2000. MR.1776072 (2001c:65001)

[22] Z. Zhang, On the hp finite element method for the one dimensional singularly perturbed convection-diffusion problems, Journal of Computational Mathematics 20 (2002), 599-610. MR1938640 (2003j:65071)

Department of Mathematics, Wayne State University, Detroit, Michigan 48202

E-mail address: zzhang@math.wayne.edu 